\title{
Alpha-Band Phase Synchrony Is Related to Activity in the Fronto-Parietal Adaptive Control Network
}

\author{
Sepideh Sadaghiani, ${ }^{1}$ René Scheeringa, ${ }^{2}$ Katia Lehongre, ${ }^{3}$ Benjamin Morillon, ${ }^{3}$ Anne-Lise Giraud, ${ }^{3}$ Mark D'Esposito, ${ }^{1}$ \\ and Andreas Kleinschmidt ${ }^{4}$ \\ ${ }^{1}$ Helen Wills Neuroscience Institute and Department of Psychology, University of California Berkeley, Berkeley, California 94720, ${ }^{2}$ Donders Institute for \\ Brain Cognition and Behaviour, Centre for Cognitive Neuroimaging, Radboud University Nijmegen, 6500 HB Nijmegen, The Netherlands, ${ }^{3}$ INSERM Unité \\ 960, Laboratoire de Neurosciences Cognitives, Ecole Normale Supérieure, 75005 Paris, France, and ${ }^{4}$ Department of Clinical Neurosciences, University \\ Hospital (HUG) and University of Geneva, CH-1211 Geneva, Switzerland
}

Neural oscillations in the alpha band $(8-12 \mathrm{~Hz})$ are increasingly viewed as an active inhibitory mechanism that gates and controls sensory information processing as a function of cognitive relevance. Extending this view, phase synchronization of alpha oscillations across distant cortical regions could regulate integration of information. Here, we investigated whether such long-range cross-region coupling in the alpha band is intrinsically and selectively linked to activity in a distinct functionally specialized brain network. If so, this would provide new insight into the functional role of alpha band phase synchrony. We adapted the phase-locking value to assess fluctuations in synchrony that occur over time in ongoing activity. Concurrent EEG and functional magnetic resonance imaging (fMRI) were recorded during resting wakefulness in 26 human subjects. Fluctuations in global synchrony in the upper alpha band correlated positively with activity in several prefrontal and parietal regions (as measured by fMRI). fMRI intrinsic connectivity analysis confirmed that these regions correspond to the well known fronto-parietal (FP) network. Spectral correlations with this network's activity confirmed that no other frequency band showed equivalent results. This selective association supports an intrinsic relation between large-scale alpha phase synchrony and cognitive functions associated with the FP network. This network has been suggested to implement phasic aspects of top-down modulation such as initiation and change in moment-to-moment control. Mechanistically, long-range upper alpha band synchrony is well suited to support these functions. Complementing our previous findings that related alpha oscillation power to neural structures serving tonic control, the current findings link alpha phase synchrony to neural structures underpinning phasic control of alertness and task requirements.

\section{Introduction}

Oscillations in the near $10 \mathrm{~Hz}$ range are the single most salient feature in the electroencephalogram (EEG) of ongoing human brain activity. These so-called alpha oscillations were traditionally considered an idling rhythm that gives way to other neural processes when sensorimotor or cognitive challenges arise (Berger, 1929). More recent studies have established that the presence of alpha oscillations exerts an overall inhibitory effect on cortical processing, but also that such inhibition may be actively induced by cognitive control mechanisms and that it can improve behavioral performance (Haegens et al., 2010, Klimesch et al., 2007).

In a separate line of research, it has been established that the power of alpha oscillations as indexed by EEG is intrinsically

\footnotetext{
Received March 19, 2012; revised Aug. 16, 2012; accepted Aug. 17, 2012.

Author contributions:S.S. designed research; S.S. performed research; K.L., B.M., and A.-L.G. contributed unpublished reagents/analytic tools; S.S. analyzed data; S.S., R.S., M.D., and A.K. wrote the paper.

This work was funded by the Agence Nationale de la Recherche, France (Grant SPONTACT). S.S. is supported by the Deutsche Forschungsgemeinschaft (Germany). We thank Kristopher Anderson and Esther Aarts for helpful discussions.

Correspondence should be addressed to Sepideh Sadaghiani, University of California Berkeley, 132 Barker Hall, D’Esposito Laboratory, Berkeley, CA 94720. E-mail: sepideh.sadaghiani@gmail.com.

DOI:10.1523/JNEUROSCI.1358-12.2012

Copyright $\odot 2012$ the authors $\quad 0270-6474 / 12 / 3214305-06 \$ 15.00 / 0$
}

coupled to activity in different functional brain networks as recorded in concurrent functional magnetic resonance imaging (fMRI). During task-free "resting-state" studies, spontaneous fluctuations in alpha oscillation power correlate negatively with activity in the so-called dorsal attention system (DAT) of superior frontal and intraparietal regions (Laufs et al., 2003; Mantini et al., 2007), but positively with activity in a cingulo-opercular (CO) network comprising dorsal anterior cingulate cortex, frontal operculum/anterior insula, and thalamus, which has been related to sustained cognitive control and maintaining alertness (Dosenbach et al., 2007; Sadaghiani et al., 2010).

alpha oscillations play a prominent role in the control of cortical excitability. Mechanistically, the phase of alpha oscillations corresponds to cyclic excitability changes. Increased alpha power will induce greater cyclic inhibition, and alpha power decreases a release from this inhibition (Haegens et al., 2011). In selective attention, spatially specific alpha power decrease yields enhanced cortical excitability and hence increased activity gain (Rajagovindan and Ding, 2011). Accordingly, neural and perceptual responses to stimuli are facilitated during reduced alpha power (Romei et al., 2008) and modulated by the alpha phase at which stimulation occurs (Busch et al., 2009; Mathewson et al., 2011; Scheeringa et al., 2011). We have metaphorically alluded to the functional consequences of this pulsed inhibition as a "wind- 
shield wiper" effect, and have suggested that its benefit in the context of cognitive and attentional demands lies in regular clearance and updating of incoming information (Sadaghiani et al., 2010). From that perspective, it is immediately intuitive that not just the local power but also the synchronization of alpha oscillations across distant cortical regions by phase locking should be functionally important, as also suggested in proposals by Palva and Palva (2007). Phase locking of alpha oscillations across cortical regions could temporally align segregated chunks of information in a way that facilitates, for instance, multisensory and sensorimotor integration or more complex perception-action loops.

Based on our earlier approaches to these issues, we therefore pursued here the question of whether the manifestation of such long-range cross-region coupling in the alpha band is intrinsically and selectively linked to activity in a distinct functionally specialized brain network. If so, this would provide some evidence that phase locking is functionally associated with those specific cognitive operations involving this network. We recorded concurrent EEG and fMRI during task-free resting state and measured synchrony by adapting the phase-locking value (PLV) (Lachaux et al., 1999) to quantify variations in ongoing phase lag over time in a sliding-window procedure. We found that phase locking in the upper alpha band is selectively associated with activity in a well defined intrinsic functional connectivity network (ICN). This network comprises frontal and parietal lobe regions, is involved in cognitive control, and is distinct from the networks associated with global alpha power.

\section{Materials and Methods}

Subjects and data acquisition. We used the same dataset in which activity correlations with alpha oscillations power were established previously (Sadaghiani et al., 2010) (including details on data acquisition and fMRI preprocessing). This allowed for direct comparison of the results. Twenty-six healthy volunteers (age 19-29 years; 7 females; 5 were left handed; all gave written informed consent) underwent simultaneous EEG (62-channel Easycap) and fMRI (Tim-Trio, Siemens). Subjects were requested to rest with eyes closed, stay awake, and avoid movement. Three $10 \mathrm{~min}$ sessions of blood oxygenation level-dependent (BOLD) fMRI ( 2 s resolution) and continuous EEG ( $5 \mathrm{kHz}$ resolution) plus an anatomical image were recorded.

EEG preprocessing. Gradient and pulse artifact subtraction are described by Sadaghiani et al. (2010). Data were downsampled to $250 \mathrm{~Hz}$ and notch filtered at 20,40, and $50 \mathrm{~Hz}$ (line and fMRI noise). As in the analysis by Lachaux et al. (1999), recordings were rereferenced to a common average reference, and the original reference electrode was recalculated as FCz.

Determining EEG oscillation band of interest. To test our hypothesis regarding upper alpha oscillations (Sadaghiani et al., 2010), we determined this band subject by subject. The power spectrum $(1-50 \mathrm{~Hz})$ was calculated using a multitaper approach in Fieldtrip (http://www.ru.nl/ fcdonders/fieldtrip) ( $2 \mathrm{~s}$ windows incremented in $0.4 \mathrm{~s}$ steps). For each subject and session, the frequency with the peak power value across all electrodes (i.e., global field power) between 8 and $12 \mathrm{~Hz}$ was identified (average $9.4 \pm 0.6 \mathrm{~Hz}$ ). The individual upper alpha band was determined as the range from individual alpha peak frequency to $2 \mathrm{~Hz}$ above (Klimesch et al., 2007).

EEG PLV time course calculation. We calculated phase synchrony over time to measure its spontaneous continuous fluctuations. First, data were bandpass filtered to the individual upper alpha band. The order of the finite-impulse-response bandpass filter was chosen to cover four cycles of the individual upper alpha band.

To calculate PLVs continuously over time rather than across trial repetitions, the formula introduced by Lachaux et al. (1999) was adapted as follows:

$$
\mathrm{PLV}=\frac{1}{N} *\left|\Sigma_{n=1}^{N} \exp \left[i\left(\phi_{\text {channel1 }}(n)-\phi_{\text {channel2 }}(n)\right)\right]\right|
$$

where $N=$ number of time points in a time window, and |.| represents the complex modulus. We used Hilbert transformation to calculate the analytic phase at time point $n$ as $\phi_{\text {channel_ } x}(n)=\arctan (u(n) / v(n))$, where $v$ is the real part of the analytic signal, $u$ is the Hilbert transform or the imaginary part of the analytic signal. For a given channel pair, the difference in phase was calculated at each time point. PLV was calculated within a $10 \mathrm{~s}$ window sliding in $2 \mathrm{~s}$ steps and hence resulting in a PLV time course sampled at $0.5 \mathrm{~Hz}$. Each PLV value reflects the consistency of phase lag between two channels across $10 \mathrm{~s}$. The PLV time course was then averaged across all channel pairs, resulting in a global PLV time course, and was converted to $z$-scores. As artifact removal strategy, extreme $z$-scores $>4$ (i.e., PLVs $>4$ SDs off the mean) were replaced by 0 , effectively removing one to five single PLVs for eight of the subjects. The remaining values were again $z$-transformed, which effectively implies that the values categorized as potential artifacts were replaced by the mean.

For several canonical frequency bands $(\delta=1-3 \mathrm{~Hz}, \theta=4-7 \mathrm{~Hz}$, low alpha $=8-9.5 \mathrm{~Hz}, \beta=13-25 \mathrm{~Hz}$, low $\gamma=26-45 \mathrm{~Hz}$ ), bandpass filtering and PLV time-course calculations were conducted accordingly.

Calculation of the imaginary part of PLV. To investigate the results without putative contributions from volume conductance, we additionally calculated the absolute value of the imaginary part of PLV for the upper alpha band (similar to imaginary coherence previously defined for coherence measures by Nolte et al., 2004):

$$
\operatorname{ImPLV}=\frac{1}{N} *\left|\operatorname{Im}\left(\sum_{n=1}^{N} \exp \left[i\left(\phi_{\text {channel1 }}(n)-\phi_{\text {channel } 2}(n)\right)\right]\right)\right| .
$$

While the imaginary part of PLV is not affected by volume conduction and common reference effects, it is sensitive to changes in the angle between two signals, which do not necessarily imply a PLV change. Correlations with this measure are therefore not unequivocally related to PLV. Conversely, spatial congruence of effects related to the imaginary part with those observed for the PLV provide a strong argument that PLV correlations are not solely due to volume conduction and reference effects.

Voxelwise regression of $f M R I$ signal onto PLV time course. Here, we sought to map the brain regions whose activity covaried with the spontaneous fluctuations in upper alpha band phase synchrony. The upper alpha global PLV time course was entered into a general linear model (GLM) as a parametric modulation regressor using SPM8 (www.fil.ion. ucl.ac.uk). Hereby, the PLV time course was convolved with the canonical hemodynamic response function (HRF). Signals of all white matter, gray matter, CSF, and out-of-brain voxels as well as the motion parameters served as nuisance covariates. First-level contrast images corresponding to the parametric regressor were entered into a second level one-sample $t$ test. An auxiliary uncorrected threshold of $p<0.005$ and extent of $>100$ voxels was used. Results are reported at $p<0.05$, cluster level corrected.

$f M R I$ intrinsic functional connectivity. For spatial comparison of the PLV-correlated activity pattern with the fronto-parietal (FP) ICN, the latter was defined using seed-based connectivity analysis as described by Sadaghiani et al. (2010). The 6-mm-radius spheres centered at standardized stereotactic coordinates $\pm 43,22,34$ (Dosenbach et al., 2007) of left and right dorsolateral prefrontal cortex (dlPFC) were joined into one seed.

Network-based regression of PLV time course onto fMRI signal. To test for frequency specificity of the observed relation to the FP network, the PLV time courses were regressed onto the FP ICN fMRI signal for several frequency bands. The BOLD time course was extracted from the FP ICN [group-level contrast, $p<0.01$ familywise error (FWE) corrected, restricted to subject-specific gray matter] and averaged across all the voxels of the network into a single mean time course. For each channel-pair a separate GLM was constructed with the respective HRF-convolved and $z$-transformed PLV time course. We included as nuisance covariates the signals of all white-matter, gray-matter, CSF, and out-of-brain voxels. 


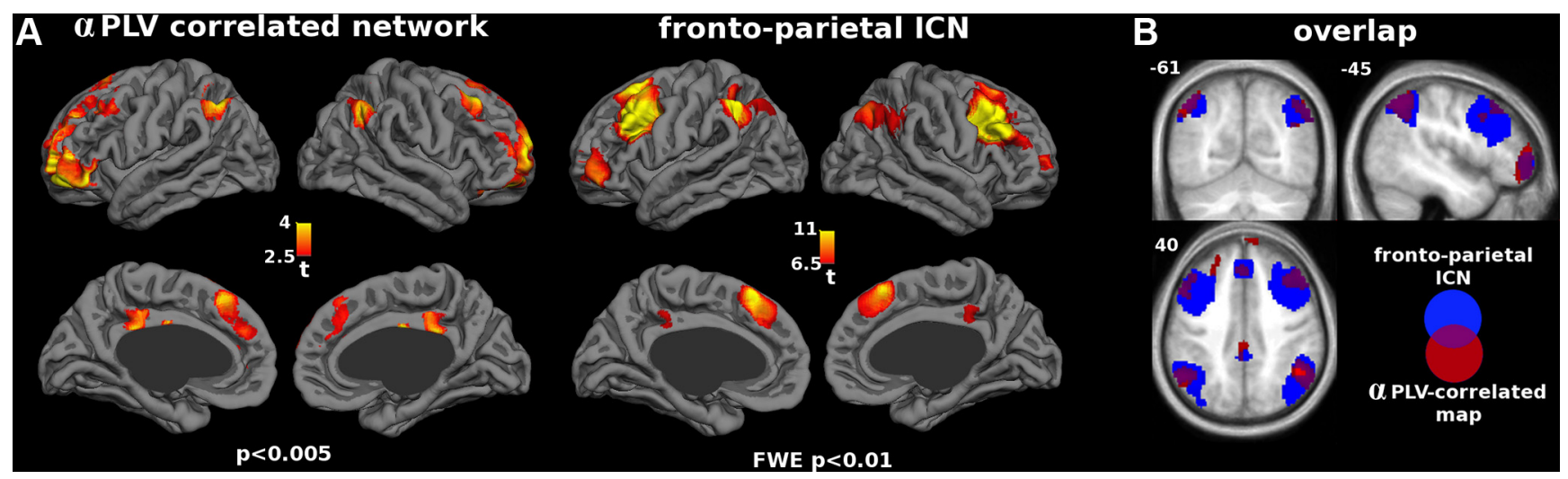

Figure 1. Brain activity correlated with global PLV time course in the upper alpha band. $A$, Left, Whole-brain regression of BOLD signal onto upper alpha band PLV time course. $p<0.005$ uncorrected, voxel extent $>100$. Right, FPICN with seed in dIPFC. $p<0.01$, FWE corrected. $\boldsymbol{B}$, Overlap of maps from $\boldsymbol{A}$ and $\boldsymbol{B}$ demonstrated on average brain slices.

This yielded for each channel pair a $\beta$-value for the PLV regressor, which was tested at group level using a one-sample $t$ test against zero. False discovery rate (FDR) statistics were applied on the corresponding $p$ values correcting for multiple comparisons $(q=5 \%$, independent or positively correlated values assumed).

Quantification of volume overlap. We assessed overlap between the PLVcorrelated map and ICNs of interest. Each map was gray matter masked (group average mask), making them more comparable. The percentage of voxels in the PLV-correlated map (uncorrected $p<0.005$, extent $>100$ voxels) overlapping with the respective ICN was calculated (ICNs defined at FWE-corrected $p<0.01$, extent $>150$ voxels). For comparability, results were normalized to the size of the respective ICN (volume of DAT and CO ICNs relative to the FP ICN: 0.85 and 1.5, respectively).

\section{Results}

Spontaneous fluctuations in global upper alpha phase synchrony during a "resting state" were significantly correlated to changes of ongoing activity in a bilateral network of prefrontal and parietal regions (Fig. 1A, left). This network comprised right and left angular gyrus/inferior parietal lobe (IPL; [46, - 52, 38], 896 voxels, peak $z$-score $=4.13$; and $[-44,-58,50], 689$ voxels, peak $z$-score $=3.52)$, right anterior ventrolateral PFC (vlPFC; [26, 64, $10], 2205$ voxels, $z=3.79)$, right dlPFC ([38, 28, 50], 1026 voxels, $z=3.60)$, and a large cluster including left anterior vlPFC ([ -38 , $54,-12], z=4.13)$, left dlPFC $([-28,32,42], z=3.11)$, and bilateral paracingulate cortex $([-8,26,50], z=3.85)$. There was a trend ( $>200$ voxels) at uncorrected level in middle cingulate cortex (mCC; [2, -36, 30], 232 voxels, $z=3.46$ ).

The spatial pattern derived from alpha PLV resembles an ICN of frontal and parietal regions previously suggested to underlie adaptive or executive control (Dosenbach et al., 2007, Seeley et al., 2007). We therefore mapped this FP ICN in our fMRI dataset using seed-based resting-state functional connectivity analyses. In accordance with the aforementioned studies, the resulting network comprised, in addition to the bilateral dlPFC seeds, regions in vlPFC, paracingulate cortex, IPL, and mCC (Fig. $1 A$, right). An overlap map (Fig. $1 B$ ) shows that there is high correspondence between the FP ICN and the PLV-correlated network from Figure $1 A$. Qualitatively, all brain regions whose activity is correlated to the alpha PLV time course directly correspond to a region of the FP ICN and vice versa. Direct quantification of this overlap is difficult due to the different nature of their defining methods. Nevertheless, a comparison with the ICNs related to alpha band power (Sadaghiani et al., 2010) is informative about the selectivity of the observed correspondence; $43 \%$ of the PLV-correlated volume overlapped with the FP ICN, while only 3 and 5\%, respectively, overlapped with the CO and DAT ICNs.

We addressed potential artifacts in an additional analysis. While PLV is less subject to contamination by amplitude effects than coherence-based synchrony measures, it can be prone to artificial phase locking due to volume conductance and common reference (Nolte et al., 2004). In the additional analysis, we therefore restricted the PLV calculation to the imaginary part. The imaginary part of the PLV is only sensitive to non-zero-phase lags and thus is resistant to instantaneous "self-interaction" from volume conductance. Using this imaginary part of PLV as a regressor, we again observed a significant correlation with fluctuations in a network comprising right and left IPL, right and left anterior vlPFC, and right dlPFC. Left dlPFC split into two regions (183 and 185 voxels) and thus did not reach cluster-level significance (Fig. 2). These results are highly congruent with the network observed using the PLV as regressor. This therefore suggests that the observed relation between the FP network and oscillation phase locking was not substantially contaminated by artificial phase synchrony.

To assess whether this relation was specific to the alpha band, we reversed the direction of regression. For upper alpha and several canonical frequency bands, the PLV time course of each channel pair was regressed onto the BOLD signal time course extracted from the FP ICN (Fig. 3). At the group level, after FDR correction for multiple comparisons 430 channel pairs passed corrected threshold for upper alpha band [number of expected false positives (5\%) 22]. Strong PLV-fMRI correlation was observed beyond the electrode pairs at short distance along the diagonal. Note, however, that the precise topography may be strongly subject to the effects of common referencing. There were no values passing corrected significance for any other band. This strongly supports the selectivity of the effect for upper alpha band.

\section{Discussion}

Large-scale phase synchronization of brain oscillations has been proposed as a key concept in neural processes underlying cognition (Varela et al., 2001; Wang, 2010). Regarding the alpha band, empirical support for this notion comes from studies that have reported enhanced long-range alpha phase synchrony across diverse cognitive paradigms ranging from low- to high-level perception, from mental imagery to working memory, especially when top-down control demands are increased (Doesburg et al., 
2009, Mima et al., 2001, Palva et al., 2010, Sauseng et al., 2005, von Stein et al., 2000, Zanto et al., 2011). Here, we complement these behavioral correlations with alpha synchrony by correlations obtained from concurrent fMRI recordings. We demonstrate a selective intrinsic link between variations in global upper alpha band phase synchrony and localized brain activity in a well defined top-down control network.

Our results are in apparent disagreement with a similar previous study. Jann et al. (2009) reported a correlation between global alpha phase synchrony and activity in the so-called default mode network. Possible explanations for this discrepancy are a different measure of phase synchrony and a fixed rather than individually determined high alpha range across subjects in that study. Their results might therefore correspond to functionally different oscillations than ours. Regarding our approach based on the PLV, we qualitatively replicated the results in an additional analysis with the imaginary part of the PLV and thus corroborated that our findings were not substantially contaminated by artificial biases of the synchrony measure. Moreover, we established that phase synchrony in no other canonical frequency band exhibited a similar corre-

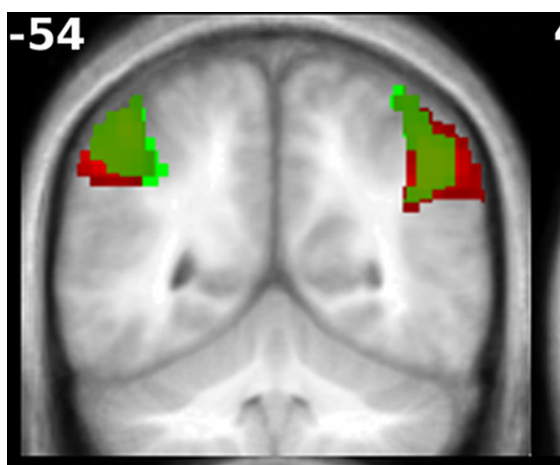

44

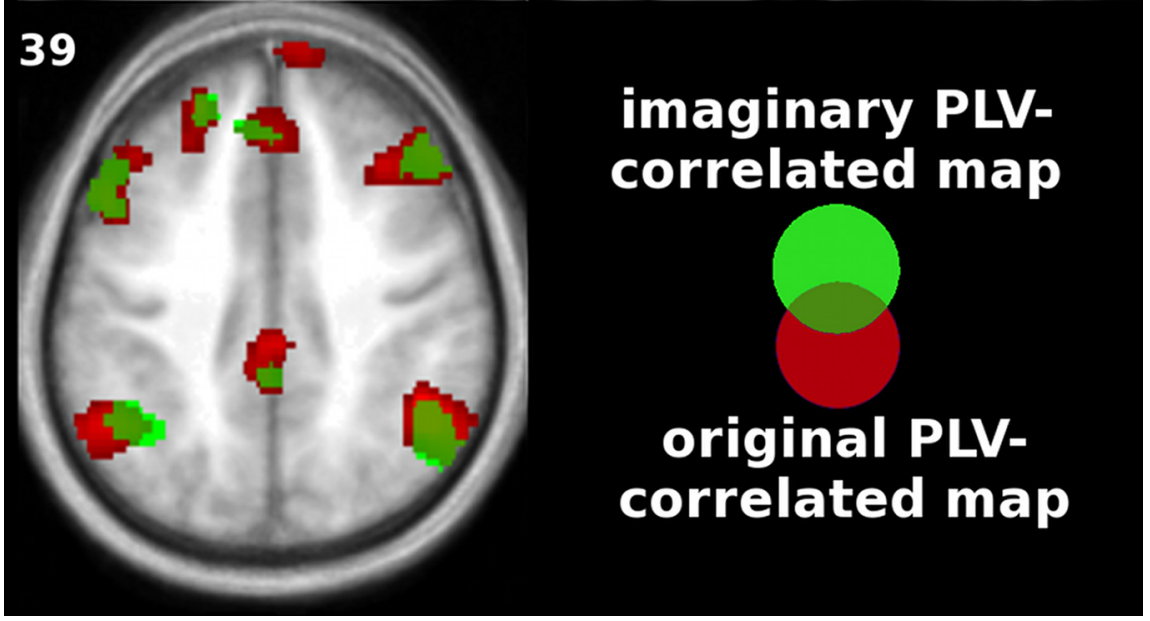

Figure 2. Overlap of results using PLV versus the imaginary part only. Whole-brain regression of BOLD signal onto upper alpha band PLV time course results in similar networks when using PLV and the imaginary part. For illustration purposes, $p<0.01$ uncorrected, voxel extent $>50$. lation with FP network activity. This se-

lective and specific relation of alpha phase synchrony to activity of the FP network suggests an intrinsic association of large-scale alpha phase locking with cognitive operations supported by this network.

The intrinsic connectivity network showing the positive correlation with alpha synchrony is one of many task-positive networks involved in attentional and cognitive control functions. Previous findings disagreed as to whether the CO and FP networks form two distinct networks (Dosenbach et al., 2007, Nomura et al., 2010, Seeley et al., 2007) or one unitary network (Vincent et al., 2008). Our previous findings within the same dataset of correlation of alpha amplitude selectively with the $\mathrm{CO}$ network favor the existence of two functionally segregated systems (Sadaghiani et al., 2010). The current data further support this view by showing a dissociation that ties activity in the FP network to large-scale alpha phase synchrony. Hence, the CO and FP networks are distinguished not only by different intrinsic functional connectivity patterns (Dosenbach et al., 2007, Seeley et al., 2007) but also by different electrophysiological signatures, involving either global power (CO ICN) or long-range synchrony (FP ICN) of alpha oscillations.

Functions of these two networks seem equally dissociable. The $\mathrm{CO}$ network has been implicated in sustained task-related cognitive control (Dosenbach et al., 2007) and maintenance of tonic alertness (Sadaghiani et al., 2010). The FP network is suggested to support phasic aspects of cognitive control such as exogenously triggered initiation of control, adapting of control for instance after errors (Dosenbach et al., 2007) and moment-to-moment executive control as in repeated rapid task switching (Seeley et al., 2007). From a more perceptual perspective, these functions read- ily correspond to a distinction in psychological literature (Posner and Boies, 1971), namely between tonic (and intrinsically maintained) alertness and phasic (and externally triggered) alertness. Phasic and adaptive control requires fast long-range communication and integration. As discussed above, alpha phase synchrony is very well suited for this function, and it is conceivable that such phasic control can be recruited by bottom-up (salience) as well as top-down mechanisms. alpha phase synchrony between lower-level regions could permit forming temporary neural assemblies, hence facilitating information integration (e.g., between unimodal sensory areas, Mima et al., 2001), and in turn could be subject to top-down modulation by FP regions. Alternatively, synchrony could underpin vertical integration between lower-level regions and executive higher-order areas (e.g., across occipito-parietal and prefrontal cortices, Zanto et al., 2011). One example of processes subject to such phasic modulation could be the integration and on-line maintenance of task-relevant information, i.e., working memory. Of note, dlPFC activity has been strongly implicated in working memory (D'Esposito, 2007), as has large-scale alpha phase synchrony (Sauseng et al., 2005; Palva et al., 2010; Zanto et al., 2011).

Another task-positive network that can be identified based on its intrinsic connectivity in resting fMRI data - the DAT - shows yet another electrophysiological signature (Laufs et al., 2003). This again can be taken as evidence for functional specialization. Figure 4 summarizes these findings for the three networks. DAT activity correlates negatively with the power of alpha oscillations. This network is commonly associated with spatial attention shifts or other manifestations of selective attention. It engages when functional benefits over and above those from sustained alertness 
A

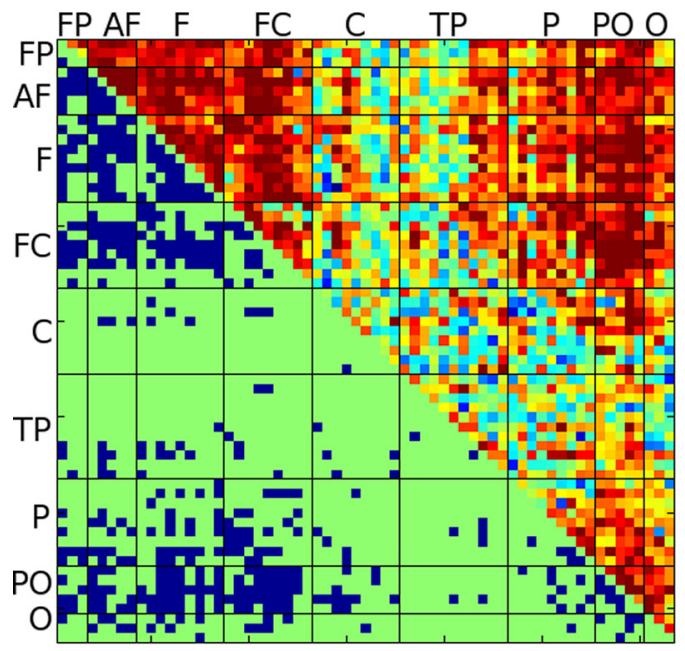

B
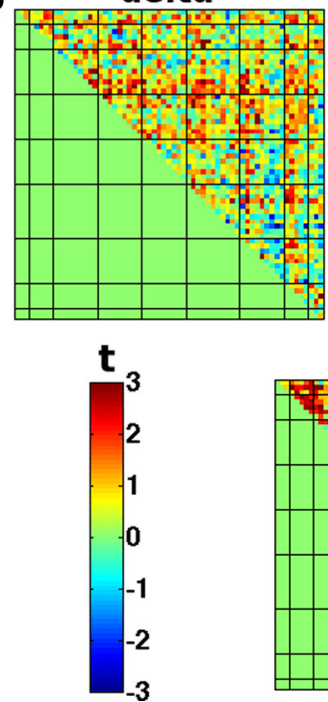

theta

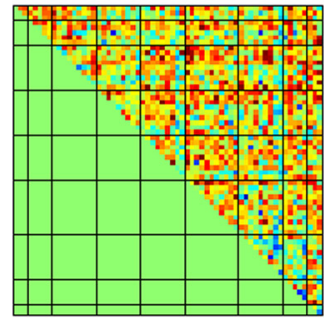

beta

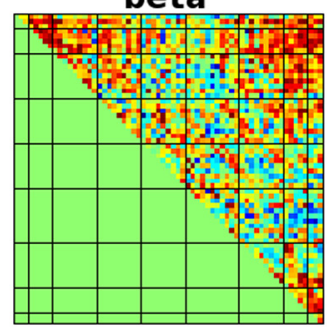

low alpha

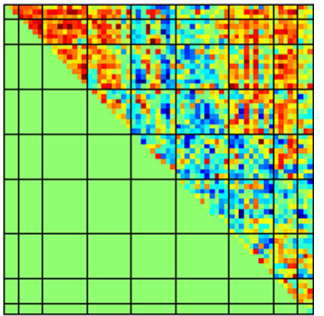

gamma

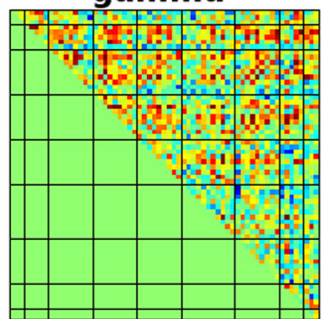

Figure 3. Group-level tstatistics demonstrating spectral specificity. $A, B$, Significant correlations (marked in blue in lower triangles) between PLV time course and BOLD time course of the FP ICN were observed for the upper alpha band $(\boldsymbol{A})$ but for no other frequency band $(\boldsymbol{B})$. Electrodes are organized frontal to occipital.

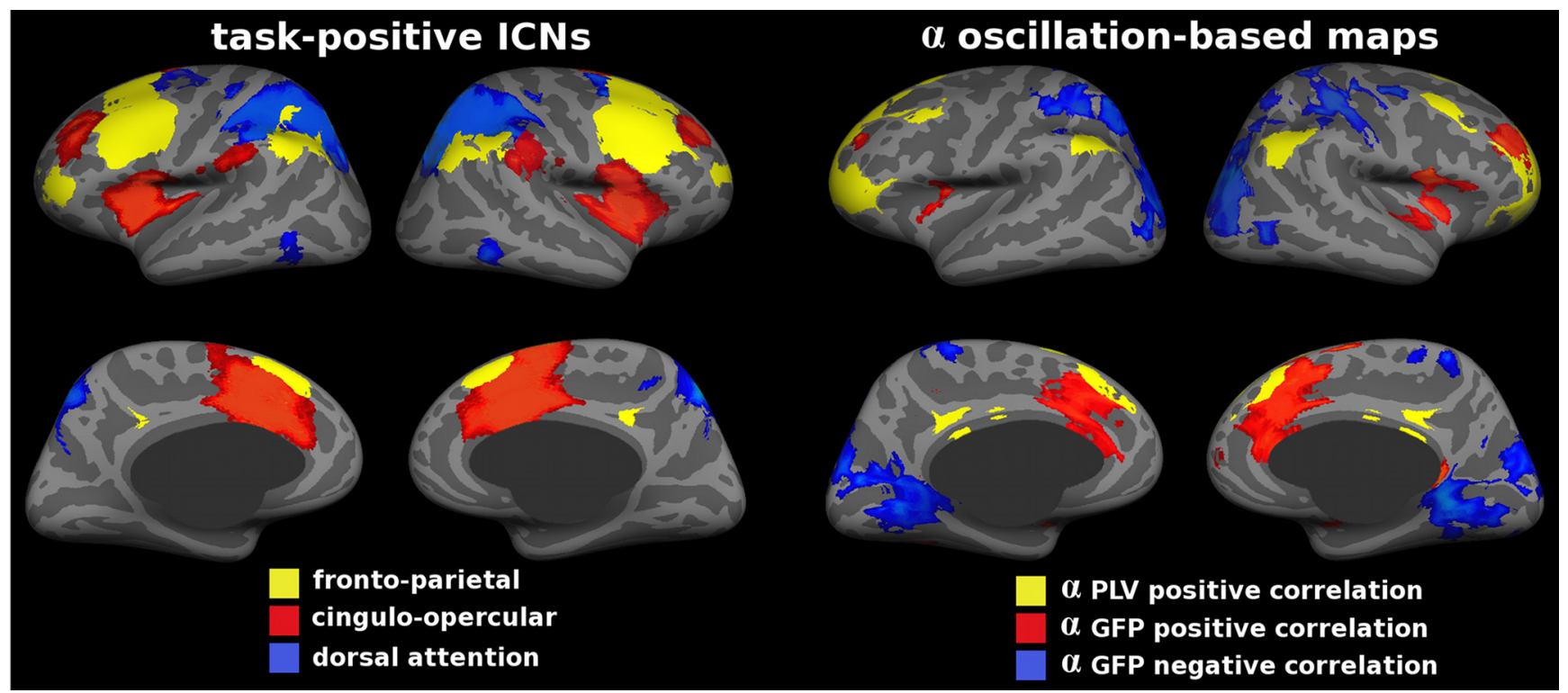

Figure 4. Summary of the selective relation between alpha oscillations and cognitive control networks. All maps are derived from the same dataset. DAT and CO ICNs (seeded in right intraparietal sulcus and right anterior insula, respectively) and maps of correlations with alpha band global field power (GFP) are taken from Sadaghiani et al. (2010). ICNs: FWE $p<0.01$, with additional voxel extent $>150$ for DAT and C 0 networks; alpha related networks: uncorrected $p<0.005$, voxel extent $>100$.

can be derived from prior expectations and guide the selective allocation of attention (e.g., to locations, features). We have previously proposed that while $\mathrm{CO}$ network activity and global alpha oscillation power underpin the sustained maintenance of nonselective alertness, DAT network activity reflects top-down guidance of selective attention. Expressed in terms of the "windshield wiper" metaphor, this corresponds to alertness driving alpha oscillations for general clearance of distracting noisy information and selective attention switching off this process selectively within relevant sensory channels (Sadaghiani et al., 2010).

In terms of connectivity and function, the DAT is often contrasted with the so-called ventral attention (VAT) network that is sensitive to unexpected salient stimuli and is proposed to draw attention to as yet unattended stimuli or locations (Corbetta and Shulman, 2002). These functions are similar to those of the FP network that is suggested to activate when initiation of top-down control is required (Dosenbach et al., 2007). There is also substantial anatomical overlap between these two networks, the main difference being that the VAT network is thought to be right lateralized (Corbetta and Shulman, 2002, Fox et al., 2006) while the FP network is bilateral (Dosenbach et al., 2007, Seeley et al., 2007). However, it has been suggested that right lateralization in attention studies might be explained by use of nonsemantic rather than meaningful stimuli (Cabeza et al., 2008). Whether the VAT largely corresponds to a specialized right-lateralized part of the FP network or constitutes a fundamentally different network in anatomy and function requires future investigation.

In conclusion, we have established that the differentiation of three important functional brain networks by virtue of different intrinsic connectivity is paralleled by distinct electrophysiological 
signatures. While all networks show selective associations with alpha oscillations, these differ in sign (DAT, CO) and in property, power versus phase locking (CO, FP). The likely reason for these associations is not direct and biophysical, but indirect and functional. We propose that different aspects of a single oscillation band may be related to the different functions of these networks and that there is a hierarchical relationship involving a role of alpha oscillations in tonic alertness, of their long-range phase locking in phasic alertness, and of their focal desynchronization in selective attention. These neurofunctional observations may help in eventually sharpening mechanistic descriptions of such concepts as tonic versus phasic alerting and alertness versus attention.

\section{References}

Berger H (1929) Über das elektroenkephalogramm des menschen. Arch Psychiatr Nervenkr 87:527-570.

Busch NA, Dubois J, VanRullen R (2009) The phase of ongoing EEG oscillations predicts visual perception. J Neurosci 29:7869-7876. CrossRef Medline

Cabeza R, Ciaramelli E, Olson IR, Moscovitch M (2008) The parietal cortex and episodic memory: an attentional account. Nat Rev Neurosci 9:613-625. CrossRef Medline

Corbetta M, Shulman GL (2002) Control of goal-directed and stimulusdriven attention in the brain. Nat Rev Neurosci 3:201-215. Medline

D'Esposito M (2007) From cognitive to neural models of working memory. Philos Trans R Soc Lond B Biol Sci 362:761-772. CrossRef Medline

Doesburg SM, Green JJ, McDonald JJ, Ward LM (2009) From local inhibition to long-range integration: a functional dissociation of $\alpha$-band synchronization across cortical scales in visuospatial attention. Brain Res 1303:97-110. CrossRef Medline

Dosenbach NU, Fair DA, Miezin FM, Cohen AL, Wenger KK, Dosenbach RA, Fox MD, Snyder AZ, Vincent JL, Raichle ME, Schlaggar BL, Petersen SE (2007) Distinct brain networks for adaptive and stable task control in humans. Proc Natl Acad Sci U S A 104:11073-11078. CrossRef Medline

Fox MD, Corbetta M, Snyder AZ, Vincent JL, Raichle ME (2006) Spontaneous neuronal activity distinguishes human dorsal and ventral attention systems. Proc Natl Acad Sci U S A 103:10046-10051. CrossRef Medline

Haegens S, Osipova D, Oostenveld R, Jensen O (2010) Somatosensory working memory performance in humans depends on both engagement and disengagement of regions in a distributed network. Hum Brain Mapp 31:26-35. Medline

Haegens S, Nácher V, Luna R, Romo R, Jensen O (2011) $\alpha$-Oscillations in the monkey sensorimotor network influence discrimination performance by rhythmical inhibition of neuronal spiking. Proc Natl Acad Sci U S A 108:19377-19382. CrossRef Medline

Jann K, Dierks T, Boesch C, Kottlow M, Strik W, Koenig T (2009) BOLD correlates of EEG $\alpha$ phase-locking and the fMRI default mode network. Neuroimage 45:903-916. CrossRef Medline

Klimesch W, Sauseng P, Hanslmayr S (2007) EEG $\alpha$ oscillations: the inhibition-timing hypothesis. Brain Res Rev 53:63-88. CrossRef Medline

Lachaux JP, Rodriguez E, Martinerie J, Varela FJ (1999) Measuring phase synchrony in brain signals. Hum Brain Mapp 8:194-208. CrossRef Medline

Laufs H, Krakow K, Sterzer P, Eger E, Beyerle A, Salek-Haddadi A, Kleinschmidt A (2003) Electroencephalographic signatures of attentional and cognitive default modes in spontaneous brain activity fluctuations at rest. Proc Natl Acad Sci U S A 100:11053-11058. CrossRef Medline
Mantini D, Perrucci MG, Del Gratta C, Romani GL, Corbetta M (2007) Electrophysiological signatures of resting state networks in the human brain. Proc Natl Acad Sci U S A 104:13170-13175. CrossRef Medline

Mathewson KE, Beck DM, Fabiani M, Ro T, Gratton G (2011) Pulsed out of awareness: EEG $\alpha$ oscillations represent a pulsed-inhibition of ongoing cortical processing. Front Percept Sci 2:99. CrossRef Medline

Mima T, Oluwatimilehin T, Hiraoka T, Hallett M (2001) Transient interhemispheric neuronal synchrony correlates with object recognition. J Neurosci 21:3942-3948. Medline

Nolte G, Bai O, Wheaton L, Mari Z, Vorbach S, Hallett M (2004) Identifying true brain interaction from EEG data using the imaginary part of coherency. Clin Neurophysiol 115:2292-2307. CrossRef Medline

Nomura EM, Gratton C, Visser RM, Kayser A, Perez F, D’Esposito M (2010) Double dissociation of two cognitive control networks in patients with focal brain lesions. Proc Natl Acad Sci U S A 107:12017-12022. CrossRef Medline

Palva JM, Monto S, Kulashekhar S, Palva S (2010) Neuronal synchrony reveals working memory networks and predicts individual memory capacity. Proc Natl Acad Sci U S A 107:7580-7585. CrossRef Medline

Palva S, Palva JM (2007) New vistas for $\alpha$-frequency band oscillations. Trends Neurosci 30:150-158. CrossRef Medline

Posner MI, Boies SJ (1971) Components of attention. Psychol Rev 78:391408. CrossRef

Rajagovindan R, Ding M (2011) From prestimulus alpha oscillation to visual-evoked response: an inverted-U function and its attentional modulation. J Cogn Neurosci 23:1379-1394. CrossRef Medline

Romei V, Brodbeck V, Michel C, Amedi A, Pascual-Leone A, Thut G (2008) Spontaneous fluctuations in posterior alpha-band EEG activity reflect variability in excitability of human visual areas. Cereb Cortex 18:2010-2018. Medline

Sadaghiani S, Scheeringa R, Lehongre K, Morillon B, Giraud AL, Kleinschmidt A (2010) Intrinsic connectivity networks, $\alpha$ oscillations, and tonic alertness: a simultaneous electroencephalography/functional magnetic resonance imaging study. J Neurosci 30:10243-10250. CrossRef Medline

Sauseng P, Klimesch W, Doppelmayr M, Pecherstorfer T, Freunberger R, Hanslmayr S (2005) EEG $\alpha$ synchronization and functional coupling during top-down processing in a working memory task. Hum Brain Mapp 26:148-155. CrossRef Medline

Scheeringa R, Mazaheri A, Bojak I, Norris DG, Kleinschmidt A (2011) Modulation of visually evoked cortical fMRI responses by phase of ongoing occipital $\alpha$ oscillations. J Neurosci 31:3813-3820. CrossRef Medline

Seeley WW, Menon V, Schatzberg AF, Keller J, Glover GH, Kenna H, Reiss AL, Greicius MD (2007) Dissociable intrinsic connectivity networks for salience processing and executive control. J Neurosci 27:2349-2356. CrossRef Medline

Varela F, Lachaux JP, Rodriguez E, Martinerie J (2001) The brainweb: phase synchronization and large-scale integration. Nat Rev Neurosci 2:229-239. CrossRef Medline

Vincent JL, Kahn I, Snyder AZ, Raichle ME, Buckner RL (2008) Evidence for a frontoparietal control system revealed by intrinsic functional connectivity. J Neurophysiol 100:3328-3342. CrossRef Medline

von Stein A, Chiang C, König P (2000) Top-down processing mediated by interareal synchronization. Proc Natl Acad Sci U S A 97:14748-14753. CrossRef Medline

Wang XJ (2010) Neurophysiological and computational principles of cortical rhythms in cognition. Physiol Rev 90:1195-1268. CrossRef Medline

Zanto TP, Rubens MT, Thangavel A, Gazzaley A (2011) Causal role of the prefrontal cortex in top-down modulation of visual processing and working memory. Nat Neurosci 14:656-661. CrossRef Medline 